The model is used to assess the 20 year impact (on HIV incidence/prevalence) of scaling up early ART to PWID, compared to a baseline of current ART access $(20 \%$ of HIV + individuals with $\mathrm{CD} 4<350$ cells $/ \mu \mathrm{L}$ are recruited onto ART annually). An uncertainty analysis was undertaken using posterior model fits to consider which PWID behavioural/epidemiological factors affect impact.

Results Only small reductions in PWID HIV incidence/prevalence ( $<10 \%$ over 20 years) will occur with current ART provision in Manipur. If current ART recruitment rates are maintained, but all HIV + PWID become eligible for ART, then a median $12 \% / 11 \%$ relative decrease in HIV incidence/prevalence occurs over 20 years compared to baseline. This increases to $22 \% / 20 \%$ or $33 \% / 28 \%$ if $40 \%$ or $80 \%$ of HIV+ PWID are recruited per year, respectively. If the ART LTFU amongst PWID is halved to $7.5 \%$ per year then impact increases by half. Uncertainty analyses suggest the impact achieved through scaling-up ART is highly dependent on baseline HIV prevalence and the cofactor increase in HIV infectivity during early infection, with less impact occurring for higher HIV prevalences and cofactors. The injecting cessation rate and level of mixing between risk groups are also important.

Discussion HIV treatment could result in large reductions in PWID HIV incidence, but is unlikely to result in local elimination except in low HIV prevalence settings.

\section{SO4.3 ANTIRETROVIRAL THERAPY AMONG FEMALE SEX WORKERS IN BURKINA FASO: CURRENT SITUATION}

doi:10.1136/sextrans-2013-051184.0027

II Konate, 'A Ouédraogo, 'WW Bazié, ${ }^{2} \mid \mathrm{T}$ Traoré, 'A Sanon, 'C Huet, ${ }^{1},{ }^{2} \mathrm{~N}$ Méda, ${ }^{3} \mathrm{P}$ Mayaud, ${ }^{4} \mathrm{P}$ Van De Perre, ${ }^{4} \mathrm{~N}$ Nagot. 'UR_SR/VIH/TB, Centre MURAZ, Bobo-Dioulasso, Burkina Faso; ${ }^{2}$ Centre de Recherche International en Santé/UO, Ouagadougou, Burkina Faso; ${ }^{3}$ LSHTM, London, UK; ${ }^{4}$ Université de Montpellier, INSERM U 1058, Montpellier, France

Background In Burkina Faso, female sex workers (FSW) are a core group in HIV transmission with $16.5 \%$ cases of HIV-infection, vs. $1.0 \%$ in the general population. Provision of ART to this core group may be key to controlling the HIV epidemic. We aimed at documenting the current situation regarding ART provision to FSW in Burkina Faso.

Methods We reviewed the interventions of the national HIV/ AIDS control programme (NACP) regarding ART provision to FSW, using grey literature and interviews of stakeholders. In addition, we reviewed the findings of the local research on this topic.

Results Before 2010, programmes targeting FSW in Burkina Faso focused only on prevention, mainly through local NGOs. From 2010, in each of the 13 regions, the NACP designated one primary health centre to care for FSW. No statistics are available yet from these centres, but $\mathrm{NACP} / \mathrm{MOH}$ stakeholders admitted that few FSW adhere to it, mainly because of a lack of strategy to reach and retain FSW, and lack of health care workers training. The ANRS 1222 "Yerelon" research programme tested a FSW peer-led intervention integrating prevention and care activities within one dedicated centre in Bobo-Dioulasso. Beside care providers, peers and a psychologist much contributed to support ART adherence before and after initiation. Preliminary data among the 47 FSW treated with ART showed high adherence to ART (> 95\%) at 12 months $(92 \%)$ and 36 months (100\%). At 36 months, plasma viral load was undetectable in $82 \%$ of FSW, with a mean CD4 increase of 230 cells $/ \mu \mathrm{L}$ (IOR: 90-400).

Conclusion Despite a specific programme, ART provision to FSW remains limited in Burkina Faso. The 'Yerelon' model of prevention and care intervention can serve as a strategy for the NACP FSW programme, and the research sites be used as training centres to improve ART provision coverage and efficiency.

\section{SO4.4 ART AMONG FSW IN SOUTH AFRICA}

doi:10.1136/sextrans-2013-051184.0028

F Venter. Wits Reproductive Health and HIV Institute (WRHI), Johannesburg, South Africa

Until recently South Africa has targeted its HIV interventions towards the general population, but the National Strategic Plan of 2012-2106 has expanded this focus to include key populations with high HIV prevalence and incidence. Foremost in this group are sex workers who are identified in the 2011 South African Know Your Epidemic (KYE) as contributing as much as $20 \%$ of the HIV transmission burden in the country. A model of health care delivery through an inner city sex worker programme has steadily expanded over a decade from special services delivered in a clinic, to clinical outreach services delivered within brothels and a mobile clinic, that now includes ART. The intervention includes behaviour change communication delivered through peer educators and outreach staff, programme-supported free clinical services to detect and treat STIs, HIV counselling and testing (HCT), TB detection, contraceptive provision, male and female condom provision. Referral linkages to relevant state services including HIV and TB treatment services and reproductive health services are in place with outreach workers tracing defaulting patients. We will discuss the outcomes of community based ART for sex workers, the challenges of provision, and present outcomes including virological suppression and loss to follow up data.

\section{S04.5 ART AMONG FSW IN INDIA}

doi:10.1136/sextrans-2013-051184.0029

\section{S Mishra, M Boily. Imperial College, London, UK}

In India, the provision of antiretroviral treatment (ART) began in 2004. Around the same time, a large-scale, condom-based HIV prevention programme targeted to FSWs was implemented in several districts. In this talk, we present findings from a comprehensive review of available data exploring trends in the ART "care-cascade" among FSWs in India, with a focus on routine HIV testing, linkage to pre-ART care, ART uptake, and retention on ART. We then examine the association between local condom-based targeted HIV prevention programmes and HIV testing among FSWs, and identify key data gaps in the "care cascade" among FSWs. We conclude by exploring the interaction between turn-over in sex work and ART eligibility among FSWs, and discuss the potential implications for local ART programmes.

\section{SO4.6 EXAMINING THE POPULATION-LEVEL IMPACT OF SCALING- UP ART FOR FSWS ACROSS EPIDEMIC CONTEXT}

doi:10.1136/sextrans-2013-051184.0030

\section{Boily, S Mishra. Imperial College, London, UK}

In this presentation, we will summarise the results of a recent systematic review of the published literature on the key parameters of the treatment cascade among female sex workers. Using different examples from transmission dynamics modelling studies of HIV transmission and ART use, we will discuss the potential impact of scaling-up ART for FSWs in contrasting epidemiological settings. We will present results of the impact of ART programmes in settings with different HIV prevalence levels in the general population (very low, low/medium, high) and different history of condom use interventions and level of ART scale-up already achieved. For example, we will compare the impact of different ART scale-up scenarios and eligibility criteria in districts of India (low prevalence settings) where there has been and there has not been a large-scale condom use intervention implemented in recent years. Other settings with 
higher HIV prevalence such as Benin, Burkina Faso, Columbia or Kisumu will be considered. We will also contrast the impact of ART programme targeted to FSW on HIV transmission in the general population and assess the influence of key parameters of the treatment cascade on the impact of ART among FSWs.

\section{S.05 - Fostering an Integrated Approach to the Control of Antimicrobial Resistance in Neisseria gonorrhoeae (WHO Symposium)}

\section{S05.1 ANTIMICROBIAL RESISTANCE IN N. GONORRHOEAE: UPDATE OF THE SITUATION}

doi:10.1136/sextrans-2013-051184.0031

T C Wi. Department of Reproductive Health Research, Controlling Sexually Transmitted Infections, World Health Organization, Geneva, Switzerland

Background The rapidly changing antimicrobial susceptibility of $N$. gonorrhoeae since the introduction of antibiotics has created challenges in gonorrhoea control. Antimicrobial resistance has regularly appeared and expanded with every release of new classes of antibiotics for gonorrhoea.

Methods The Gonococcal Antimicrobial Surveillance Programme (GASP) has been documenting the emergence and spread of antimicrobial resistance in gonorrhoea since 1992 and has informed treatment guidelines.

Results Sixty two countries participated in GASP in varying degrees including the extend of specific antimicrobials tested for resistance in 2010 . There are high rates of resistance in $N$. gonorrhoeae to penicillin and quinolones. There are 36 countries reporting increasing minimum inhibitory concentration (MIC) to Cefixime $(\geq$ $0.25 \mu \mathrm{g} / \mathrm{mL}$ ) or Ceftriaxone ( $\geq 0.125 \mu \mathrm{g} / \mathrm{mL}$ ). Treatment failures to Ceftriaxone were reported in Japan, Austria, Australia, Canada, France, Norway, Slovenia, Sweden, UK and South Africa. Majority of reports is from developed countries. This is only the tip of the global health burden as surveillance data from resource-constrained settings are scarce.

Spectinomycin and azithromycin resistance are monitored in limited countries. Brunei, China, Mongolia and Russia reported decreased susceptibility to spectinomycin. Resistance to azithromycin has been identified in some European countries, US and Chile. Azithromycin resistance remains well below the $5 \%$ threshold in most countries.

Conclusions Treatment options for gonorrhoeae is dwindling. In the short term, options for treatment will include increasing dosage, using intramuscular or intravenous route and multiple doses of Ceftriaxone; use of alternative medication (e.g. gentamycin), and combination therapies. In the long term there is a need to develop newer classes of antibiotic. There is a need to ensure rational drug use and strengthen antimicrobial resistance monitoring in gonorrhoea including detection and management of treatment failure.

\section{S05.2 CHALLENGES AND OPPORTUNITIES: POTENTIALS IN RESEARCH ON AMR GONOCOCCI}

doi:10.1136/sextrans-2013-051184.0032

C Deal. National Institute of Allergy and Infectious Diseases, Bethesda, MD, United States

Although gonorrhoea has afflicted humans for centuries and the causative bacterium, Neisseria gonorrhoeae, was identified over a century ago, gonorrhoea remains a global public health problem. Stepwise acquisition of genetic mutations has conferred gradually increasing resistance to multiple antibiotics, thus limiting their usage for presumptive therapy. Until recently cephalosporins have remained the foundation of treatment, but growing evidence suggests that resistance is emerging. Even as researchers work to understanding the genetic mutations required for cephalosporin resistance, it is clear that new treatment options are needed. This presents a challenge as the number of new systemic antimicrobials evaluated and approved each year by regulatory agencies has steadily fallen over the past 30 years. Currently one new antimicrobial is undergoing clinical study as a potential treatment of gonorrhoea. An alternative strategy is to repurpose older antibiotics by studying the efficacy of dual therapy combinations of existing antimicrobials. Ultimately new antimicrobial development is needed now, since the development process can take more than a decade.

Detecting and responding to emergence of multidrug resistant gonorrhoea remains a challenge. Rapid detection of resistant infections is facilitated by local antimicrobial susceptibility testing, which requires live organisms isolated by culture. However, as the use of nucleic acid amplification tests (NAATs) have expanded, the number of $N$. gonorrhoeae cultures performed by public health laboratories decreased rapidly and the capacity of laboratories to perform culture has declined. An alternative strategy is to develop molecular assays for detecting genetic mutations associated with resistance or susceptibility to specific antimicrobials to guide antibiotic selection by the clinician at the point of care. Even with this potential, molecular assays may not be able to supplant culture-based antimicrobial susceptibility testing for surveillance to detect novel resistance phenotypes and genotypes. Ultimately a gonococcal vaccine may be the most effective public health strategy.

\section{S05.4 INTEGRATING ANTIMICROBIAL SUSCEPTIBILITY MONITORING IN NEISSERIA GONORRHOEAE TO BROADER AMR AGENDA}

doi:10.1136/sextrans-2013-051184.0033

'M Ghidinelli, 'P Ramon-Pardo, ${ }^{2} \mathrm{~J}$ A Dillon, ${ }^{3} \mathrm{P}$ Galarza. ${ }^{1}$ Pan American Health Organization/ World Health Organization, Washington, DC, United States; ${ }^{2}$ University of Saskatchewan, Saskatoon, SK, Canada; ${ }^{3}$ NEI ANLIS Dr. Carlos G. Malbran, Buenos Aires, Argentina

Background Based upon the strategies of the WHO "Global action plan to control the spread and impact of antimicrobial resistance in Neisseria gonorrhoeae", a plan was developed and implemented to align GASP related activities within the broader agenda of the Antimicrobial Resistance Latin-America Network. Spite of the achievements of GASP in the last decades, there was a need to increase the number of countries and institutions collaborating in N. gonorrhoeae susceptibility surveillance. In order to achieve this, a comprehensive plan to strengthen the capacity of the Latin-America National Reference Laboratories (NRL) to conduct AMR surveillance in a systematic and regular manner to ensure the early detection of resistant N. gonorroheae was implemented.

Methods After a number of communications and training activities with the NRL, N. gonorrhoeae susceptibility 2010 data were collected through a questionnaire sent to the National Reference Laboratories in Latin America. Data included country, number of isolated tested, and percentage of resistance to cefixime, ceftriaxone, ciprofloxacin, spectinomycin, penicillin, and tetracycline.

Results From 21 Latin American countries integrating the network, 8 (38\%) provide data. None of them detected decreased susceptibility to ceftriaxone; resistance to ciproloxacine ranked from $0 \%$ to $83 \%$; penicillin from 6 to $78 \%$; no resistance to spectinomycin; resistance to tetracyline from 27 to $83 \%$.

\section{Conclusions}

- The NRL demonstrated certain capacity and commitment to collect, analyse, and report data on N. gonorrhoeae susceptibility, however, this should be strengthned and expanded to more countries in the Region.

- Broad differences among countries were observed, specially on the susceptibility to ciprofloxacine and penicillin. 